\title{
Permutation-Based Transmissions in Ultra-Reliable and Low-Latency Communications
}

\author{
Yuli Yang, Senior Member, IEEE
}

\begin{abstract}
In this paper, a novel permutation-based transmission strategy is proposed to improve the goodput in wireless networks for achieving ultra-reliable and low-latency communications. The proposed scheme divides the application-layer data into two portions: the first one is conveyed by the permutation with repetition of various lengths in a group of packets rather than encapsulated into the packets, whilst the second portion is encapsulated into these packets to be physically delivered through network interface in the conventional way. The lengths of the packets used to deliver the second portion are determined by the first one. Thanks to the goodput gain attained by the permutation-conveyed application-layer data, the network congestion is alleviated, which leads to lower latency and/or less dropped packets. The validity of this transmission strategy is substantiated by the analysis in the metrics of goodput, latency, physical-layer throughput and secrecy rate within a design paradigm of short-packet communications.
\end{abstract}

Index Terms-Application-layer data, data unit (DU), goodput, latency, permutation-conveyed DU (PCDU), physical-channel throughput, secrecy rate.

\section{INTRODUCTION}

As a key technology pillar in emerging wireless networking infrastructure, the ultra-reliable and low-latency communication (URLLC) with massive connectivity over scarce physical resource units is indispensable to the applications and services in the Internet of Things, the tactile Internet and vehicular networks. In practice, the URLLC features of the end-to-end connectivity need to be addressed by the whole system, from upper-layer protocols to the network interface [1].

The application-layer throughput of a communication link is defined as goodput, which is used to measure the number of application-layer data in bits delivered through the given network interface. These data exclude the overhead bits that are added in lower layers for control signalling. However, the dynamics of wireless channels leave a dilemma in the design of upper-layer protocols, i.e., the delivery of control signalling will ensure high reliability but increase the latency [2]. Besides, the network congestion due to higher traffic load and the erratic propagation due to wireless connections yield more retransmissions and/or dropped packets, which further impedes low latency in achieving high goodput.

Against this backdrop, we address the URLLC from the transport-layer perspective and propose a permutation-based transmission strategy to increase the goodput in a straightforward way. Over the past decade, the philosophy of permutation modulation has inspired the physical-layer community with the invention of spatial modulation and index modulation [3] [6], where a portion of information bits is mapped onto the

Y. Yang is with the School of Engineering, University of Lincoln, Lincoln LN6 7TS, U.K. (e-mail: yyang@ lincoln.ac.uk). resource activation in space, frequency or time domain to improve the spectral efficiency.

In this work, the permutation philosophy is embraced by the transport layer, where a portion of application-layer data is conveyed by the permutation with repetition of various lengths in a group of packets. The permutation-based transmission inherits the merits of permutation modulation in the physical layer and offers four attractive features: increased goodput, reduced latency, improved physical-channel throughput, and enhanced security against data tracing.

\section{Permutation-Based Transmissions}

In this section, the permutation-based transmission strategy is proposed and two design criteria are developed.

\section{A. System Model}

In the transport layer, connection-oriented, end-to-end reliable protocols, e.g, Transmission Control Protocol (TCP) and User Datagram Protocol (UDP), provide full access to a variety of multi-network applications. A transport-layer packet is composed of a header and a data unit (DU). The former carries the information fields formatted to handle sending/accepting the packet and managing the flow control. The length in bytes of a packet is specified by a field in the header, which indicates the DU length in this packet given that the header length is fixed [7], [8].

For the purpose of reliable transport, the TCP includes sequence number in the header to identify the order of data bytes sent from each transmitter [7]. In general, the UDP does not support retransmissions [8]. However, in specific applications of UDP for reliable transport, e.g., [9], packet numbers are used to order packets in retransmissions.

Our permutation-based transmission strategy is conceived on the basis of these two explicitly transmitted quantities, namely the DU length and the order of a packet.

Consider a group of $N$ packets, denoted by vectors $\mathbf{s}_{1}, \mathbf{s}_{2}, \cdots, \mathbf{s}_{N}$, where the $n^{\text {th }}$ packet $\mathbf{s}_{n}=\left[\mathbf{u}_{n}, \mathbf{v}_{n}\right]$ with $\mathbf{u}_{n}$ and $\mathbf{v}_{n}$ denoting the header and the DU in this packet, $n=1,2, \cdots, N$. The lengths of the DUs $\mathbf{v}_{1}, \mathbf{v}_{2}, \cdots, \mathbf{v}_{N}$ are varied, and each length is chosen from a set of $K$ values in bits denoted by $\left\{L_{0}, L_{1}, \cdots, L_{K-1}\right\}$.

Hence, there are $K^{N}$ permutations with repetition in the assignment of $K$ lengths into a group of $N$ DUs, which can be exploited to convey extra application-layer data. Elaborating slightly further, in addition to the data encapsulated into these $N$ DUs, the number of extra data conveyed by the permutation of $K$ lengths in this DU group is expressed in bits as

$$
T_{\mathrm{e}}=N \log _{2} K,
$$




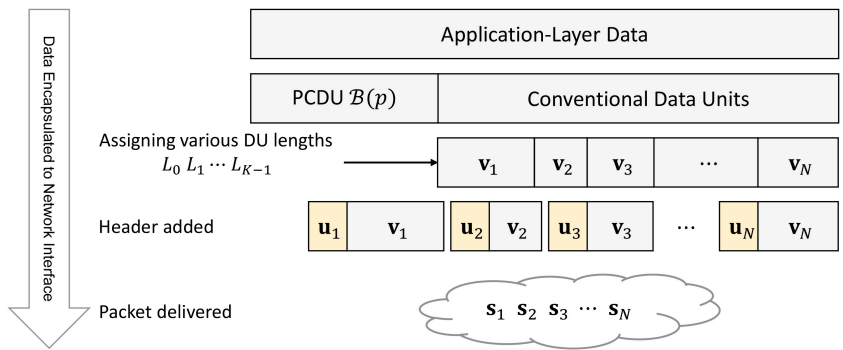

Fig. 1. The encapsulation of application-layer data in a permutation-based transmission.

where $T_{\mathrm{e}}$ denotes the number of extra application-layer data bits in a permutation-based transmission.

In our permutation-based transmission, the application-layer data to be delivered by a group of DUs is divided into two portions. The first portion, composed of $T_{\mathrm{e}}$ bits, is conveyed by the permutation of various DU lengths in this DU group and, therefore, referred to as permutation-conveyed DU (PCDU). The second portion is encapsulated into the conventional DUs $\mathbf{v}_{n}, n=1,2, \cdots, N$, whose lengths are determined by the first portion, i.e., PCDU, and physically delivered through the network interface. The encapsulation of application-layer data in a permutation-based transmission is shown in Fig. 1, where the PCDU pertaining to the $p^{\text {th }}$ permutation is expressed as $\mathcal{B}(p)$ with $\mathcal{B}(\cdot)$ denoting a function of binary coded decimal, for $p=0,1, \cdots, K^{N}-1$. For example, if $N=3$ conventional DUs are grouped for the permutation of $K=2$ DU lengths, $L_{0}$ and $L_{1}$, the resultant $K^{N}=8$ permutations and the PCDU pertaining to them are demonstrated in Table I.

In practice, the total number of application-layer data bits is determined by the flow control and congestion control. In a TCP header, the 'window' field specifies the window size in a connection [7], which indicates the number of bytes that the receiver is currently prepared to receive, denoted by $F_{\mathrm{S}}$. Although the UDP does not use a 'window' to manage the congestion control, the total number of data bits to be transmitted is limited by the receiver's buffer size [8]. Furthermore, given the TCP/UDP header length, the maximum DU length, denoted by $L_{\max }$, can be calculated according to the 'TCP/UDP length' field in the pseudo header [7], [8].

\section{B. Design Criteria}

In a permutation-based transmission of $N$ DUs, the $K$ lengths, $L_{0}, L_{1}, \cdots, L_{K-1}$, are varied for the permutation and the probability that the $k^{\text {th }}$ length $L_{k}$ occurs in the transmission is denoted by $\gamma_{k}, k=0,1, \cdots, K-1$. The mean length of a DU in this permutation group is

$$
\bar{L}=\sum_{k=0}^{K-1} \gamma_{k} L_{k} .
$$

For simplicity, the various DU lengths can be set to $L_{k}=M k$ bits, where $M$ is a natural number and $k=$ $0,1, \cdots, K-1$. Without loss of generality, these lengths are assumed to occur in the transmission at the same probability, i.e., $\gamma_{k}=1 / K, \forall k \in\{0,1, \cdots, K-1\}$, and accordingly the mean length of a DU is $\bar{L}=M(K-1) / 2$ bits. As the total

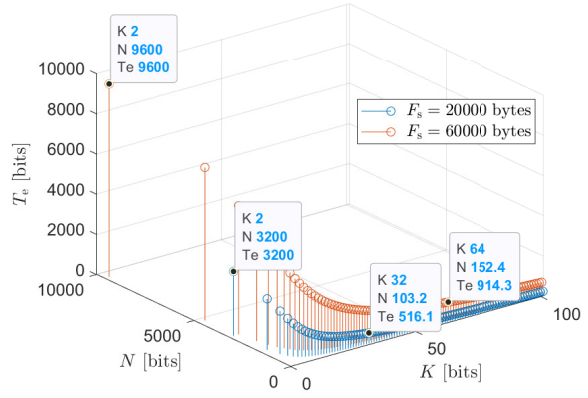

Fig. 2. $T_{\mathrm{e}}$ versus $K$ and $N$.

number of data encapsulated in the $N$ DUs is limited by the window size $F_{\mathrm{s}}$, we have $N \bar{L} \leqslant F_{\mathrm{s}}$. Moreover, the maximum DU length is limited by $L_{\max }$, i.e., $M(K-1) \leqslant L_{\max }$.

Herein, we consider two design criteria for the permutationbased transmission.

1) PCDU Length Maximisation: With this criterion, the number of DUs, $N$, and the number of DU lengths, $K$, in a permutation group are optimised to achieve the maximum PCDU length $T_{\mathrm{e}}^{*}$. Given $F_{\mathrm{s}}$ and $L_{\max }$, the maximization of $T_{\mathrm{e}}$ is formulated by an optimization problem as

$$
\begin{aligned}
& \qquad T_{\mathrm{e}}^{*}=\max _{N, K} N \log _{2} K \\
& \text { subject to } \quad 0 \leqslant N M(K-1) / 2 \leqslant F_{\mathrm{s}} \\
& \\
& 2 \leqslant K \leqslant L_{\max } / M+1
\end{aligned}
$$

where the constraints (3a) and (3b) pertain to the window size and the maximum DU length specified by the header, respectively. From (3a), we have $N \leqslant\left(2 F_{\mathrm{s}} / M\right) /(K-1)$, and then $T_{\mathrm{e}} \leqslant\left(2 F_{\mathrm{s}} / M\right) \log _{2} K /(K-1)$. With a notation $\phi(K) \triangleq \log _{2} K /(K-1)$, the maximum of $T_{\mathrm{e}}$ is rewritten as $\left(2 F_{\mathrm{s}} / M\right) \phi(K)$. Since the derivative

$$
\frac{\mathrm{d} \phi(K)}{\mathrm{d} K}=\frac{1}{(K-1)^{2}}\left(\frac{K-1}{K \ln 2}-\log _{2} K\right)<0
$$

for (3b), the function $\left(2 F_{\mathrm{s}} / M\right) \phi(K)$ is strictly decreasing given $F_{\mathrm{S}}$ and $M$. To further illustrate this function, $T_{\mathrm{e}}$ is plotted in Fig. 2 as a function of $K$ and $N$, where $T_{\mathrm{e}}$ is raised upon decreasing $K$ while increasing $N$. As a result, the solution to (3) is $T_{\mathrm{e}}^{*}=2 F_{\mathrm{s}} / M$ with the optimal design:

$$
\left\{\begin{array}{l}
K^{*}=2, \\
N^{*}=2 F_{\mathrm{s}} / M
\end{array}\right.
$$

Given the window size $F_{\mathrm{s}}$, the optimal design for maximising $T_{\mathrm{e}}$ is to vary 2 lengths in a group of $2 F_{\mathrm{s}} / M$ DUs, i.e., $L_{0}=0$ and $L_{1}=M$. This design is particularly suitable for machine-type communications, where a large number of packets are delivered but the DU in each packet is extremely short.

2) Resource Utilisation Efficiency Maximisation: With this criterion, $N$ and $K$ are optimised to maximise the resource utilisation efficiency of the permutation-based transmission, which is defined as the amount of application-layer data in total, namely the bits in the PCDU plus those in the 
TABLE I

AN EXAMPLE of 8 Permutations for $N=3$ AND $K=2$

\begin{tabular}{|c|c|c|c|c|c|c|c|c|c|c|c|c|c|c|c|c|c|c|c|c|c|c|c|c|}
\hline Permutation $\sharp$ & \multirow{2}{*}{\multicolumn{3}{|c|}{$\begin{array}{c}0 \\
000\end{array}$}} & \multirow{2}{*}{\multicolumn{3}{|c|}{$\begin{array}{c}1 \\
001\end{array}$}} & \multirow{2}{*}{\multicolumn{3}{|c|}{010}} & \multirow{2}{*}{\multicolumn{3}{|c|}{$\begin{array}{c}3 \\
011 \\
\end{array}$}} & \multirow{2}{*}{\multicolumn{3}{|c|}{$\begin{array}{c}4 \\
100\end{array}$}} & \multirow{2}{*}{\multicolumn{3}{|c|}{$\begin{array}{c}5 \\
101\end{array}$}} & \multirow{2}{*}{\multicolumn{3}{|c|}{$\begin{array}{c}6 \\
110\end{array}$}} & \multirow{2}{*}{\multicolumn{3}{|c|}{111}} \\
\hline PCDU $\mathcal{B}(p)$ & & & & & & & & & & & & & & & & & & & & & & & & \\
\hline $\mathrm{DU} \sharp$ & 1 & 2 & 3 & 1 & 2 & 3 & 1 & 2 & 3 & 1 & 2 & 3 & 1 & 2 & 3 & 1 & 2 & 3 & 1 & 2 & 3 & 1 & 2 & 3 \\
\hline DU Length & $L_{0}$ & $L_{0}$ & $L_{0}$ & $L_{0}$ & $L_{0}$ & $L_{1}$ & $L_{0}$ & $L_{1}$ & $L_{0}$ & $L_{0}$ & $L_{1}$ & $L_{1}$ & $L_{1}$ & $L_{0}$ & $L_{0}$ & $L_{1}$ & $L_{0}$ & $L_{1}$ & $L_{1}$ & $L_{1}$ & $L_{0}$ & $L_{1}$ & $L_{1}$ & $L_{1}$ \\
\hline
\end{tabular}

conventional DUs, carried by a single resource unit in the physical channel, calculated using

$$
E_{\mathrm{R}}=\frac{T_{\mathrm{e}}+N \bar{L}}{N\left(L_{\mathrm{H}}+\bar{L}\right)}=\frac{\log _{2} K+M(K-1) / 2}{L_{\mathrm{H}}+M(K-1) / 2},
$$

where $L_{\mathrm{H}}$ is the header length in bits. The maximization of $E_{\mathrm{R}}$ can be formulated by an optimization problem as

$$
\begin{aligned}
E_{\mathrm{R}}^{*}= & \max _{N, K} \frac{\log _{2} K+\bar{L}}{L_{\mathrm{H}}+\bar{L}} \\
\text { subject to } \quad & 0 \leqslant N M(K-1) / 2 \leqslant F_{\mathrm{s}} \\
& 2 \leqslant K \leqslant L_{\max } / M+1 .
\end{aligned}
$$

Apparently, $E_{\mathrm{R}}$ is a strictly increasing function of $K$, given $L_{\mathrm{H}}$ and $M$. Hence, the solution to (7) is $E_{\mathrm{R}}^{*}=\left(L_{\max }+\right.$ $\left.2 \log _{2}\left(L_{\max } / M+1\right)\right) /\left(L_{\max }+2 L_{\mathrm{H}}\right)$ with the optimal design:

$$
\left\{\begin{array}{l}
K^{*}=L_{\max } / M+1, \\
N^{*}=2 F_{\mathrm{s}} / L_{\max }
\end{array}\right.
$$

Based on the TCP header, we may have $L_{\max }=F_{\mathrm{s}}$. As such, the optimal design for maximising $E_{\mathrm{R}}$ is to vary $F_{\mathrm{s}} / M+1$ lengths in a group of 2 DUs.

In Fig. 3, the PCDU length $T_{\mathrm{e}}$ and the resource utilisation efficiency $E_{\mathrm{R}}$ are illustrated as two functions of $M$ for both design criteria, where $E_{\mathrm{R}}=(M+2) /\left(M+2 L_{\mathrm{H}}\right)$ for achieving $T_{\mathrm{e}}^{*}$ and $T_{\mathrm{e}}=2 \log _{2}\left(F_{\mathrm{s}} / M+1\right)$ for achieving $E_{\mathrm{R}}^{*}$. As is shown in this figure, larger window size $F_{\mathrm{s}}$ leads to higher goodput and resource utilisation efficiency. However, greater header length $L_{\mathrm{H}}$ reduces the resource utilisation efficiency.

We remark that, these two criteria bear on two design extremes: maximising $T_{\mathrm{e}}$ leads to the least number of DU lengths, $K$, whilst maximising $E_{\mathrm{R}}$ leads to the least number of DUs, $N$, in the permutation group. In practical applications, the tradeoff between $T_{\mathrm{e}}$ and $E_{\mathrm{R}}$ needs to be taken into account through balancing $K$ and $N$ in the design.

\section{Performance Analysis}

In this section, the performance of our permutation-based transmission strategy is investigated in the metrics of goodput, latency, physical-channel throughput and secrecy rate, within a short-packet communication paradigm.

\section{A. Metrics}

1) Goodput Gain: The total number of bits in conventional DUs delivered through the network interface in a permutationbased transmission is expressed as $T_{\mathrm{c}}=N \bar{L}$, where the mean length of a conventional DU, $\bar{L}$, is given by (2).

Subsequently, the total number of data bits, i.e., the bits in the PCDU plus those in the conventional DUs $\mathbf{v}_{n}, n=$

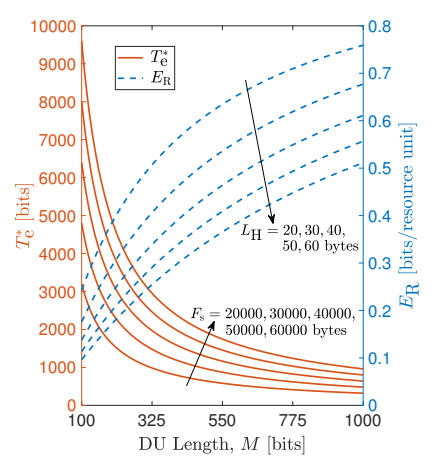

(a) $T_{\mathrm{e}}$ Maximisation $\left(K^{*}=2\right)$

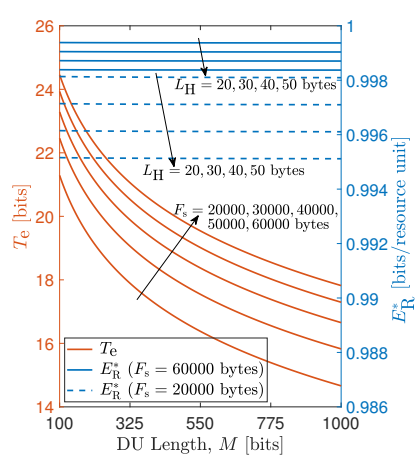

(b) $E_{\mathrm{R}}$ Maximisation $\left(N^{*}=2\right)$
Fig. 3. The tradeoff between the PCDU length $T_{\mathrm{e}}$ and the resource utilisation efficiency $E_{\mathrm{R}}$, versus the DU length difference $M$.

$1,2, \cdots, N$, delivered in a permutation-based transmission is $T_{\mathrm{e}}+T_{\mathrm{c}}$, where $T_{\mathrm{e}}$ is given by (1).

The goodput gain achieved by the permutation-based transmission, denoted by $\eta_{\mathrm{G}}$, is defined as the ratio of the number of bits in the PCDU to the number of bits in the conventional DUs, i.e.,

$$
\eta_{\mathrm{G}}=\frac{T_{\mathrm{e}}}{T_{\mathrm{c}}}=\frac{\log _{2} K}{\bar{L}}=\frac{2 \log _{2} K}{M(K-1)},
$$

which is determined by $K$ and $M$, but independent of $N$.

From (9), we may find that higher goodput gain is achieved by permutation-based transmissions of shorter packets, i.e., smaller $\bar{L}$ leads to larger $\eta_{\mathrm{G}}$. Besides, if the DU lengths $L_{0}, L_{1}, \cdots, L_{K-1}$ are fixed, activating greater lengths at lower probabilities and shorter lengths at higher probabilities will result in smaller $\bar{L}$ and thereby larger $\eta_{\text {G. That is, the goodput }}$ gain can be further improved through the source coding of application-layer data.

2) Latency: The main causes of latency in a network include traffic load and retransmissions, both of which can be alleviated by our permutation-based transmission. As shown in Fig. 1, the bits in the PCDU are not transmitted physically in the conventional way but mapped onto the permutation of various DU lengths. Thus, the PCDU neither generates any traffic load nor requires any retransmission, which guarantees successful delivery of $T_{\mathrm{c}}+T_{\mathrm{e}}$ bits through the physical transmission of $T_{\mathrm{c}}$ bits.

Concerning the network congestion state, the retransmission chance, and the data transfer rate are consistent for the long run, the ratio of the permutation-based transmission latency to the conventional transmission latency, denoted by $\eta_{\mathrm{L}}$, can be formulated using

$$
\eta_{\mathrm{L}}=\frac{T_{\mathrm{c}}}{T_{\mathrm{c}}+T_{\mathrm{e}}}=\frac{M(K-1)}{M(K-1)+2 \log _{2} K} .
$$


However, the $T_{\mathrm{e}}$ bits in the PCDU will suffer from a delay of $N$ packets if they come from a consistent bulk of applicationlayer data. As $T_{\mathrm{e}}=N \log _{2} K$, the length of a DU $\mathbf{v}_{n}$ can be determined according to the $\log _{2} K$ bits that are followed by this DU, for framing the permutation group of $N$ DUs, which will guarantee the shortest latency for the data in the PCDU.

3) Physical-Channel Throughput: This metric is defined as the effective data rate per physical channel use, in the unit of $[\mathrm{bits} / \mathrm{sec} / \mathrm{Hz}]$. The physical-channel throughput of the permutation-based transmission is calculated using

$$
R_{\mathrm{pbt}}=\frac{T_{\mathrm{c}}+T_{\mathrm{e}}}{D_{\mathrm{H}}+N L_{\mathrm{H}}+T_{\mathrm{c}}} \log _{2}(1+\rho),
$$

and that of the conventional transmission is given by

$$
R_{\text {con }}=\frac{T_{\mathrm{c}}}{D_{\mathrm{H}}+N L_{\mathrm{H}}+T_{\mathrm{c}}} \log _{2}(1+\rho),
$$

where $\rho$ is the signal-to-noise power ratio (SNR) in the physical channel, and $D_{\mathrm{H}}$ is the overhead in bits introduced by the frame header/trailer at the data link layer and the pilots at the physical layer.

4) Secrecy Rate: A physical-layer secret key can be generated by varying the bit-to-symbol mapping patterns according to the instantaneous channel quality indicator (CQI) of legitimate link [10]. Based on this concept, the mapping pattern of PCDU can be varied in terms of legitimate CQI to further enhance the security. If there are $P=K^{N}$ permutations, the number of PCDU mapping patterns is $P$ !. In the example shown in Table I, there are 8 ! mapping patterns for PCDU, denoted by $\mathfrak{M}_{q}, q=1,2, \cdots, 8$ !, and the mapping pattern elaborated in this example is $\mathfrak{M}_{1}: \sharp p \rightarrow \mathcal{B}(p)$, $p=0,1, \cdots, 7$. Another mapping pattern can be expressed as $\mathfrak{M}_{2}: \sharp p \rightarrow \mathcal{B}(p+1 \bmod 8), p=0,1, \cdots, 7$, for instance.

The PCDU mapping pattern can be deemed to be a secret key, if it is varied according to the legitimate CQI. Therefore, eavesdroppers can never recover the bits in the PCDU even if they search all possible mapping patterns in a brute-force way, since they have no basis to pick up the correct mapping for the recovery. If an eavesdropper sticks to a single mapping pattern, it may correctly recover $1 / K^{N}$ of the bits in the PCDU statistically and, however, cannot recognise which bits are correctly recovered. As such, the secrecy rate of the permutation-based transmission is achieved at

$$
R_{\mathrm{s}, \mathrm{pbt}}=\frac{T_{\mathrm{e}}}{D_{\mathrm{H}}+N L_{\mathrm{H}}+T_{\mathrm{c}}} C_{\mathrm{t}}+R_{\mathrm{s}, \mathrm{con}},
$$

where $C_{\mathrm{t}}$ denotes the achievable data rate of the legitimate link and $R_{\mathrm{s}, \mathrm{con}}$ is the secrecy rate of conventional CQI-mapped transmissions.

\section{B. A Paradigm of Short-Packet Communications}

For achieving higher goodput, short-packet communications benefit more from our permutation-based transmission strategy. Herein, we investigate a permutation-based paradigm of short-packet communications using UDP to achieve URLLC, where $F_{\mathrm{s}}=1500$ bytes, the header length $L_{\mathrm{H}}=8$ bytes, and the overhead in the frame, $D_{\mathrm{H}}=8$ bytes. Based on the optimal designs (5) and (8), the equiprobable lengths are assigned to

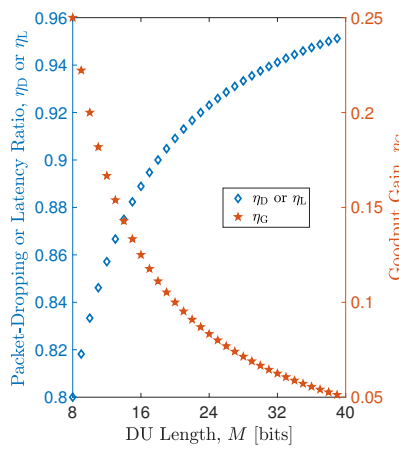

(a) $T_{\mathrm{e}}$ Maximisation

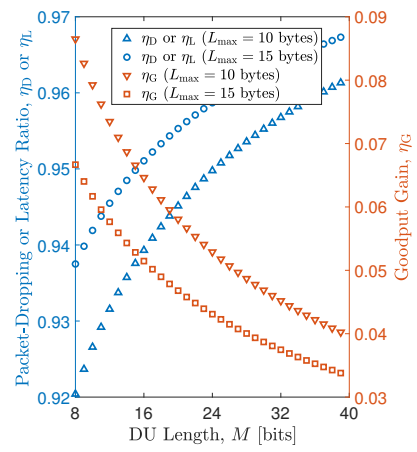

(b) $E_{\mathrm{R}}$ Maximisation
Fig. 4. The packet-dropping (or latency) ratio $\eta_{\mathrm{D}}$ (or $\eta_{\mathrm{L}}$ ) and the goodput gain $\eta_{\mathrm{G}}$, versus the DU length difference $M$.

a group of $N$ DUs. In the UDP, $T_{\mathrm{c}}=F_{\mathrm{S}}$. With the criterion of $T_{\mathrm{e}}$ maximisation, $T_{\mathrm{e}}^{*}=2 F_{\mathrm{s}} / M$. With the criterion of $E_{\mathrm{R}}$ maximisation, $T_{\mathrm{e}}=\left(F_{\mathrm{s}} / L_{\max }\right) \log _{2}\left(L_{\max } / M+1\right)$.

UDP is often used in time-sensitive applications, where dropping packets is preferable to waiting for packets delayed due to retransmission [8]. However, in specific applications for reliable transport, UDP has to support retransmissions. The contribution of our PCDU lies in reducing the traffic load, which leads to less dropped packets for the UDP without retransmission and shorter latency for the UDP with retransmission. The ratio of the packet-dropping chance in the permutation-based transmission to that in the conventional transmission, denoted by $\eta_{\mathrm{D}}$, is the same as the latency ratio $\eta_{\mathrm{L}}$ given by (10).

The packet-dropping (or latency) ratio $\eta_{\mathrm{D}}$ (or $\eta_{\mathrm{L}}$ ) and the goodput gain $\eta_{\mathrm{G}}$ of this paradigm are plotted versus the DU length difference $M$ in Fig. 4 for both criteria, where the ratios in the criterion of $T_{\mathrm{e}}$ maximisation are independent of $L_{\max }$ because the longest DU length $M \ll L_{\max }$. For the criterion of $E_{\mathrm{R}}$ maximisation, $\eta_{\mathrm{D}}$ (or $\eta_{\mathrm{L}}$ ) increases and $\eta_{\mathrm{G}}$ decreases upon increasing $L_{\max }$. As shown in this figure, the criterion of $T_{\mathrm{e}}$ maximisation has less packets dropped and achieves higher goodput gain than the criterion of $E_{\mathrm{R}}$ maximisation. In addition, shorter DU length offers less packets dropped and higher goodput gain in the permutation-based transmission.

When it comes to the physical-channel throughput, the effective data rate per physical channel use is taken into account. The impact of the DU length difference $M$ on the physical-channel throughput is investigated in Fig. 5, where the physical-channel throughput of this paradigm, $R_{\mathrm{pbt}}$, is given by (11) and that of the conventional transmission, $R_{\text {con }}$, is given by (12). For the criterion of $T_{\mathrm{e}}$ maximisation, $N=2 F_{\mathrm{s}} / M$ in (11) and (12). For the criterion of $E_{\mathrm{R}}$ maximisation, $N=2 F_{\mathrm{s}} / L_{\max }$ in (11) and (12). As shown in this figure, the physical-channel throughput of the permutation-based transmission is always higher than that of the conventional transmission. Moreover, the design for $E_{\mathrm{R}}$ maximisation achieves higher physical-layer throughput than the design for $T_{\mathrm{e}}$ maximisation. The main reason behind this is that more meta-data is introduced in the design for $T_{\mathrm{e}}$ maximisation by a large number of packets, which reduces 


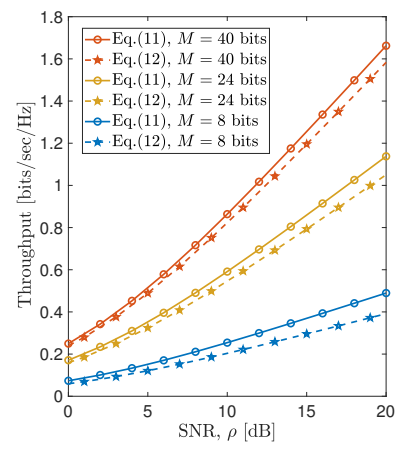

(a) $T_{\mathrm{e}}$ Maximisation

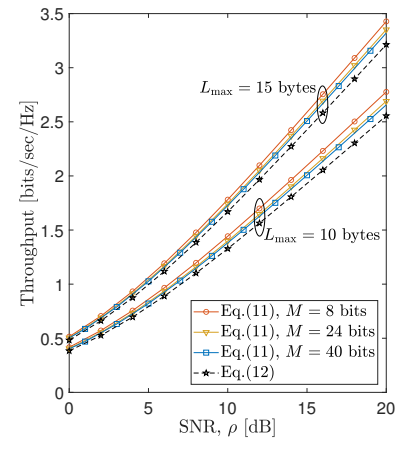

(b) $E_{\mathrm{R}}$ Maximisation
Fig. 5. The physical-channel throughput comparisons between the permutation-based transmission and the conventional transmission.

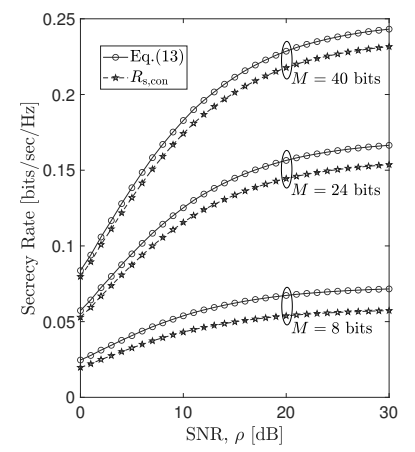

(a) $T_{\mathrm{e}}$ Maximisation

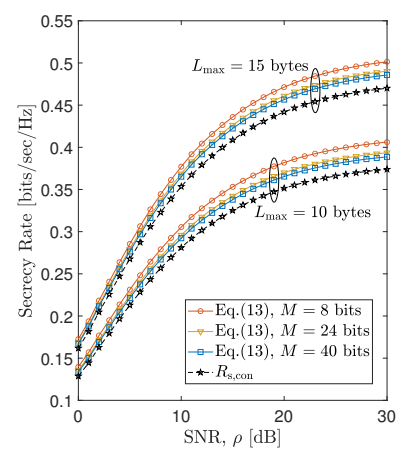

(b) $E_{\mathrm{R}}$ Maximisation
Fig. 6. Secrecy rate comparisons between the permutation-based transmission and the conventional transmission in a fading channel with BPSK modulation.

the effective throughput. Furthermore, the physical-channel throughput gap between the permutation-based transmission and the conventional transmission is reduced as $M$ increases, for both criteria, mainly because the goodput gain achieved by the permutation-based transmission decreases with the increase in $M$.

In Fig. 6, the secrecy rate of the permutation-based transmission, given by (13), is compared with that of the conventional transmission, $R_{\mathrm{s}, \text { con }}=T_{\mathrm{c}} C_{\mathrm{t}} /\left(D_{\mathrm{H}}+N L_{\mathrm{H}}+T_{\mathrm{c}}\right)$, over a fading channel with BPSK modulation, where $C_{\mathrm{t}}$ is the achievable data rate of a BPSK system, calculated using

$$
\begin{aligned}
C_{\mathrm{t}}=1-\frac{1}{2} \mathcal{E}_{\mu}\left\{\mathcal { E } _ { w } \left\{\log _{2}\left(1+e^{-\frac{2 \mu^{2}+2 \mu w}{\sigma^{2}}}\right)\right.\right. \\
\left.\left.+\log _{2}\left(1+e^{-\frac{2 \mu^{2}-2 \mu w}{\sigma^{2}}}\right)\right\}\right\}
\end{aligned}
$$

with $w \sim \mathcal{N}\left(0, \sigma^{2}\right)$ denoting the additive white Gaussian noise and $\mu \sim \mathcal{N}(0,1)$ denoting the channel fading. As shown this figure, the permutation-based transmission always achieves higher secrecy rate than the conventional transmission, and the design for $E_{\mathrm{R}}$ maximisation achieves higher secrecy rate than the design for $T_{\mathrm{e}}$ maximisation. In addition, the secrecy rate gap between the permutation-based transmission and the conventional transmission is reduced as $M$ increases, for both criteria.

\section{CONCLUSION AND Discussion}

A novel permutation-based transmission strategy was proposed in this paper for transport-layer protocols to achieve URLLC, where the permutation with repetition of various lengths in a group of DUs was exploited to convey an extra portion of application-layer data. To further quantify the performance gain achieved by the proposed scheme, the optimal designs were formulated for two criteria: PCDU length maximisation and resource utilisation efficiency maximisation. Given the window size and maximum DU length, the former criterion leads to high goodput improvement and the latter leads to highly effective throughput.

The permutation-based transmission can be flexibly compatible/coexisting with conventional encapsulation of applicationlayer data in the transport layer [7], by using one bit in the 'reserved' field to specify whether the permutation-based or the conventional encapsulation is being executed.

From the performance analysis for the optimal designs (5) and (8), we may find that the performance gain achieved by permutation-based transmissions over conventional transmissions gets higher in the permutation group of more DUs (larger $N$ ) with shorter lengths (smaller $M$ ). However, the meta-data structure in classical upper-layer protocols limits the advantage of permutation-based design. This calls for efficient encoding of control information and smart structure of meta-data, which is also a key to the coexistence of short-packet and long-packet communications in future wireless networks.

\section{REFERENCES}

[1] G. Sutton, et al., "Enabling technologies for ultra-reliable and low latency communications: From PHY and MAC layer perspectives", IEEE Commun. Surveys Tuts., vol. 21, no. 3, pp. 2488-2524, Feb. 2019.

[2] H. Shariatmadari, et al., "Fifth-generation control channel design: Achieving ultrareliable low-latency communications", IEEE Veh. Technol. Mag., vol. 13, no. 2, pp. 84-93, Jun. 2018.

[3] Y. Yang and B. Jiao, "Information-guided channel-hopping for high data rate wireless communication", IEEE Commun. Lett., vol. 12, no. 4, pp. 225-227, Apr. 2008.

[4] M. Wen, et al., "Multiple-mode orthogonal frequency division multiplexing with index modulation", IEEE Trans. Commun., vol. 65, no. 9, pp. 3892-3906, Sep. 2017.

[5] N. Ishikawa, et al., "50 years of permutation, spatial and index modulation: From classic RF to visible light communications and data storage", IEEE Commun. Surveys Tuts., vol. 20, no. 3, pp. 1905-1938, Mar. 2018.

[6] Q. Li, et al., "Diversity enhancing multiple-mode OFDM with index modulation", IEEE Trans. Commun., vol. 66, no. 8, pp. 3653-3666, Aug. 2018.

[7] J. Postel, "Transmission Control Protocol", Internet Engineering Task Force (IETF) RFC 793, Sep. 1981.

[8] J. Postel, "User Datagram Protocol", Internet Engineering Task Force (IETF) RFC 768, Aug. 1980.

[9] R. Hamilton, et al., "QUIC: A UDP-based secure and reliable transport for HTTP/2", Internet Engineering Task Force (IETF) Network Working Group Internet-Draft, Jul. 2016.

[10] Y. Yang and M. Guizani, "Mapping-varied spatial modulation for physical layer security: Transmission strategy and secrecy rate", IEEE J. Sel. Areas Commun., vol. 36, no. 4, pp. 877-889, Apr. 2018. 